\title{
Interpreting the spectral behavior of MWC $314^{\star}$
}

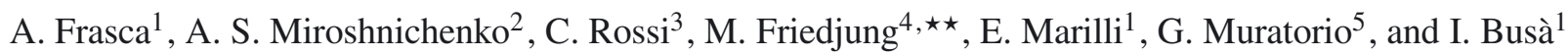 \\ 1 INAF, Osservatorio Astrofisico di Catania, via S. Sofia, 78, 95123 Catania, Italy \\ e-mail: antonio.frasca@oact.inaf.it \\ 2 Department of Physics and Astronomy, University of North Carolina at Greensboro, Greensboro, NC 27412, USA \\ 3 Department of Physics, University La Sapienza, Piazzale A. Moro 5, 00185 Roma, Italy \\ 4 Institut d'Astrophysique, CNRS, Paris 6, 75014 Paris, France \\ 5 OMP/LAM, 13004 Marseille, France
}

Received 21 July 2015 / Accepted 16 October 2015

\section{ABSTRACT}

\begin{abstract}
Context. MWC 314 is one of the most luminous stars in the Milky Way. Its fundamental parameters are similar to those of luminous blue variables (LBVs), although no large photometric variations have been recorded. Moreover, it shows no evidence of either a dust shell or a relevant spectral variability.

Aims. The main purpose of this work is to clarify the origin of the radial velocity and line profile variations exhibited by absorption and emission lines.

Methods. We analyzed the radial velocity (RV) variations displayed by the absorption lines from the star's atmosphere using highresolution optical spectra and fitting the RV curve with an eccentric orbit model. We also studied the RV and profile variations of some permitted and forbidden emission lines of metallic ions with a simple geometric model. The behavior of the Balmer and He I lines has also been investigated.

Results. Fourier analysis applied to the RV of the absorption lines clearly shows a 60-day periodicity. A dense coverage of the RV curve allowed us to derive accurate orbital parameters. The RV of the Fe II emission lines varies in the same way, but with a smaller amplitude. Additionally, the intensity ratio of the blue/red peaks of these emission lines correlates with the RV variations. The first three members of the Balmer series as well as [N II] lines display a nearly constant RV and no profile variations in phase with the orbital motion instead. The He I $\lambda 5876 \AA$ Aine shows a strongly variable profile with broad and blue-shifted absorption components that reach velocities of $\leq-1000 \mathrm{~km} \mathrm{~s}^{-1}$ in some specific orbital phases.

Conclusions. Our data and analysis provide strong evidence that the object is a binary system composed of a supergiant B[e] star and an undetected companion. The emission lines with a non-variable RV could originate in a circumbinary region. For the Fe II emission lines, we propose a simple geometrical two-component model where a compact source of Fe II emission, moving around the center of mass, is affected by a static extra absorption that originates from a larger area. Finally, the blue-shifted absorption in the He I $\lambda 5876 \AA$ line could be the result of density enhancements in the primary star wind that is flowing towards the companion, and which is best observed when projected over the disk of the primary star.
\end{abstract}

Key words. binaries: spectroscopic - stars: emission-line, Be - stars: individual: MWC 314

\section{Introduction}

There has been debate about whether MWC 314 is a B[e] supergiant or a luminous blue variable (LBV). It appears to be one of the most luminous stars in the Galaxy (Miroshnichenko et al. 1998). However, it does not show the typical brightness variations of the latter class. The profiles of the permitted emission lines show two peaks, which suggests their formation in a rotating disk, and which led us to consider MWC 314 as a $\mathrm{B}[\mathrm{e}]$ supergiant rather than an LBV (Muratorio et al. 2008).

Marston \& McCollum (2008) detected a very large bipolar nebula that emits in $\mathrm{H} \alpha$ and extends for $\sim 13.5 \mathrm{pc}$. It takes over $10^{5}$ years to form such a nebula at a typical expansion velocity for LBV candidates of $50 \mathrm{~km} \mathrm{~s}^{-1}$ (Nota et al. 1995). They propose that this star and other $\mathrm{B}[\mathrm{e}]$ supergiants with large nebulae could have passed the LBV phase and have been moving towards the blue part of the upper HR diagram. This possibility

\footnotetext{
* Based on observations made at the $0.91 \mathrm{~m}$ of Catania Observatory, the OHP telescopes and the $1.83 \mathrm{~m}$ telescope of the Asiago Observatory. $\star \star$ Michael Friedjung passed away on October 22, 2011.
}

is also supported by the absence of circumstellar dust, analogously to P Cyg, as already noted by Miroshnichenko (1996) and confirmed by the spectral energy distributions of these two stars in Lobel et al. (2013). This might mean that the dust has been blown away by the wind and one or more LBV outbursts, which occurred a long time ago.

The possibility of binarity of MWC 314 was suggested by Wisniewski et al. (2006) from fast variations of the $\mathrm{H} \alpha$ emission line strength. It was further indicated by the discovery of regular displacements of the absorption-line centroids in the spectrum of MWC 314 (Muratorio et al. 2008).

Regular radial velocity (RV) variations of four absorption lines with a period of 60.8 days were detected by Lobel et al. (2013) based on 15 high-resolution optical spectra. These authors concluded that MWC 314 is a single-lined binary system with an LBV-like early B-type supergiant primary, with a mass of $\sim 40 M_{\odot}$, which has a strong asymmetric wind. No signature of the secondary companion was found, although they suggested that it might be a less massive relatively $\operatorname{cool}\left(T_{\text {eff }} \sim 6200 \mathrm{~K}\right)$ giant. 
Richardson et al. (2014) presented a larger set of highresolution spectroscopic data that cover the period 2001-2013 and derived a slightly different orbit using the same absorption lines as Lobel et al. (2013). However, they concluded that the primary star in the system has a mass of $\sim 5 M_{\odot}$. Such a low mass was explained by a strong mass loss as a result of a transfer toward the secondary, stellar wind and eruptions that led to the mass ratio reversal. At the same time, these authors did not reconsider the primary's luminosity, thus suggesting that it is significantly overluminous for its current mass.

Richardson et al. (2014) also obtained $K^{\prime}$-band interferometry that was modelled with a point-like source plus an elliptical Gaussian corresponding to a circumbinary disk tilted by $50-65^{\circ}$ with respect to the line of sight. However, they do not rule out that this structure might be a jet roughly aligned with the $\mathrm{H} \alpha$ bipolar outflow.

In any case, the lack of detection of spectral signatures from the secondary component does not permit more accurate masses of the stellar components being established.

To study this binary system with a large set of data and to look for correlations of permitted and forbidden emission lines with the orbital phase, we performed a dense spectroscopic monitoring of MWC 314 between September 2007 and October 2009.

In this paper we describe the results obtained from the analysis of all the spectra collected from 1994 to 2009. We studied the RV behavior both of absorption and emission lines, the simultaneous ratio of blue/red peak intensities of permitted emission lines, and the total equivalent width of the emission lines.

\section{Observations and data reduction}

The first set of spectra was obtained between 1994 and 1998 at OHP with both the AURELIE and ELODIE spectrographs and one spectrum at Ekar-Asiago in 2006, with the REOSC échelle spectrograph. A detailed logbook of these observations and the data reduction procedures are described in Muratorio et al. (2008).

Additional spectra were collected between September 2007 and October 2009 in the wavelength range 4300-6850 $\AA$ at the $91 \mathrm{~cm}$ telescope of the Catania Observatory (OAC) with the FRESCO spectrograph, which gives a spectral resolving power of $R \simeq 21000$. In some cases we were able to follow the star for several consecutive nights.

The data were reduced with the ECHELLE task of the IRAF package, following standard steps. The data analysis was performed with IRAF routines and IDL ${ }^{1}$ procedures.

The log of all the spectroscopic observations is presented in Table 1, where also the numerical results of our analysis are reported.

An ELODIE spectrum showing the most prominent emission lines in the range 4300-6850 $\AA$ is shown in Fig. 1 .

\section{Data analysis}

We studied the behavior of absorption and emission (permitted and forbidden) lines from several points of view.

We used the absorption lines only to measure the RV with the aim of confirming the strong variations detected in a relatively short time (Muratorio et al. 2008) and to refine the parameters of the periodicity, which were previously found on the basis of a

\footnotetext{
1 IDL (Interactive Data Language) is a registered trademark of Exelis Visual Information Solutions.
}

small number of observations spanning a period of several years. In the hypothesis of a binary system, possibly composed of two very luminous stars, we studied the emission lines, with the aim of understanding not only their forming regions and the cause of their double-peaked shape, but also of interpreting their temporal variations and looking for any possible correlation with the RV of the absorption lines.

\subsection{Absorption lines}

All the absorption lines are very faint, therefore we carefully selected a number of them to use for the RV determination. We chose lines of the same ion and possibly same multiplet, and previously noted because their wavelength clearly varies in time (see, e.g., Rossi et al. 2011, and Fig. 2 of the present paper). The final list of these lines is as follows: S II $(\lambda 5201.0, \lambda 5212.6, \lambda 5320.7, \lambda 5453.8, \lambda 5473.6, \lambda 5556.0$, $\lambda 5564.9, \lambda 5606.1, \lambda 5640.0, \lambda 5647.0)$, N II $(\lambda 5676.0, \lambda 5679.6$, and $\lambda 5710.8)$, and $\mathrm{NeI}(\lambda 6402.2)$. A few other lines were also considered in the spectra with the highest signal-to-noise ratio $(\mathrm{S} / \mathrm{N})$.

For each line we measured the centroid wavelength and converted it into the heliocentric RV. For each spectrum we then computed the average and the standard deviation of all the individual RV values. These values are reported in Table 1.

Thanks to a large number of spectra taken in a long-term time span (from 1994 to 2009) we were able to accurately determine the period of the RV variations. For this task we used periodogram analysis (Scargle 1982) and the CLEAN deconvolution algorithm (Roberts et al. 1987), which allowed us to reject aliases generated by the spectral window of the data. We found a period of $60.8 \pm 1.7$ days (see Fig. 3). The data folded with the period display a smooth variation with an asymmetrical shape typical for an eccentric RV orbital motion (see Fig. 4a). Then, we fitted the observed RV curve with the CURVEFIT routine (Bevington 1969) to determine the orbital parameters and their standard errors. This also allowed us to improve the determination of the orbital period. The orbital parameters that result from the fit of the RV curve are presented in Table 2, and the phase computed for each observation is quoted in Col. 5 of Table 1.

Taking our 48 spectra, which span $\sim 15$ years and provide a well-phased coverage of the orbital cycle, we find orbital elements that are in a fairly good agreement with those derived by Lobel et al. (2013). Our orbital period is only $0.1 \%$ smaller, the eccentricity is $\sim 4 \%$ larger, and the RV semi-amplitude is $6 \%$ larger than those of Lobel et al. (2013). The RV errors of our data are similar to those quoted by these authors, but we derive larger errors for the orbital parameters. In particular, we find an error of $11 \mathrm{~min}$ for the orbital period, much larger than that of only $1.2 \mathrm{~s}$ reported by Lobel et al. (2013). We think that our value of the orbital period error, which is not affected by possible errors resulting from the relatively large photometric uncertainties, is more reliable.

Our orbital solution derived from the absorption lines coincides with that of Richardson et al. (2014) within the uncertainties, although our error for the orbital period is three times smaller. For a direct comparison of our results with those of Lobel et al. (2013) and Richardson et al. (2014) see Table 2.

From our values of $P, k$, and $e$, we have computed the mass function, defined as

$f(m)=\frac{m_{2}^{3}}{\left(m_{1}+m_{2}\right)^{2}} \sin i^{3}=k^{3} \frac{P}{2 \pi G}\left(1-e^{2}\right)^{\frac{3}{2}}$. 
Table 1. Radial velocity of absorption lines $\left(\mathrm{RV}_{\mathrm{abs}}\right)$, blue/red peak intensity ratio $(B / R)$, velocity of the blue and red emission peaks and radial velocity of single emissions (see text).

\begin{tabular}{|c|c|c|c|c|c|c|c|c|c|c|c|c|}
\hline Date & $\begin{array}{l}\text { HJD } \\
2400000+\end{array}$ & $\begin{array}{r}\mathrm{RV}_{\mathrm{abs}} \\
(\mathrm{km}\end{array}$ & Err & Phase & $B / R$ & Err & $\begin{array}{r}\mathrm{RV}_{\mathrm{em}}^{\mathrm{B}} \\
(\mathrm{km} \mathrm{s}\end{array}$ & Err & $\begin{array}{r}\mathrm{RV}_{\mathrm{em}}^{\mathrm{R}} \\
(\mathrm{km}\end{array}$ & Err & $\begin{array}{r}\mathrm{RV}_{\mathrm{em}}^{\mathrm{S}} \\
(\mathrm{km}\end{array}$ & Err \\
\hline 19940828 & 49593.39754 & -25 & 6 & 0.780 & 1.15 & 0.12 & -6.7 & 2.4 & 59.6 & 2.6 & 27.2 & 2.7 \\
\hline 19950607 & 49876.58716 & 97 & 4 & 0.443 & 0.55 & 0.11 & -8.2 & & 55.1 & 3.1 & 42.9 & 4.3 \\
\hline & & 47 & 5 & 0.643 & 0.87 & 0.06 & -0.6 & 3.1 & 63.7 & 2.7 & 37.4 & 3.7 \\
\hline 19980705 & 000.46328 & -80 & 7 & & $\ldots$ & $\ldots$ & $\ldots$ & $\ldots$ & $\ldots$ & $\ldots$ & $\ldots$ & $\ldots$ \\
\hline 19980721 & 1016.46947 & 95 & 9 & 0.210 & $\ldots$ & $\ldots$ & $\ldots$ & $\ldots$ & $\ldots$ & $\ldots$ & $\ldots$ & \\
\hline 19980909 & 1066.35217 & -38 & 5 & 0.032 & 1.06 & 0.04 & -2.0 & 5.7 & 59.8 & 3.1 & 34.9 & 3.5 \\
\hline 20060716 & 53933.40647 & 85 & 6 & 0.236 & 0.76 & 0.03 & -5.7 & 4.3 & 68.4 & 3.4 & 40.9 & 4.1 \\
\hline 20070903 & 54347.40815 & . & . & 0.052 & 0.77 & 0.08 & 13.1 & 2.0 & 81.0 & 3.0 & 36.4 & 3.6 \\
\hline 20070929 & 4373.38428 & 85 & 8 & 0.480 & 0.55 & 0.10 & -5.8 & 1.1 & 58.4 & 0.7 & 42.5 & 4.2 \\
\hline 20070930 & 54374.35878 & 85 & 7 & 0.496 & 0.50 & 0.12 & -8.8 & 1.6 & 57.9 & 0. & 43.1 & 4.3 \\
\hline 20080517 & 54604.50475 & 105 & 8 & 0.285 & 0.74 & 0.11 & 3.6 & 3.6 & 73.5 & 4. & 39.9 & 3.9 \\
\hline 20080521 & 54607.54430 & 102 & 1 & 0.335 & 0.66 & 0.0 & -6.2 & 2.8 & 65.4 & 1. & 43.2 & 4.3 \\
\hline 20080523 & 4609.56085 & 104 & 4 & 0.368 & 0.65 & 0.1 & -3.6 & 4.7 & 66.5 & 3. & 46.3 & 4.6 \\
\hline 20080525 & & 102 & 5 & & & 0.0 & -7.6 & & 61.6 & & 43.6 & 4.3 \\
\hline & & 94 & 6 & & & & -9.5 & & 58.9 & & 45.3 & 4.5 \\
\hline & & 12 & 8 & & & & -5.6 & & 62 & & 30.9 & 3.0 \\
\hline & & 6 & 10 & & & & -2.0 & & 0 & & 9.3 & 2.9 \\
\hline & & -11 & 4 & & 1.1 & & -7.2 & & 0 & & 2.0 & 2.2 \\
\hline 200 & 5 & 90 & 10 & 0.3 & 0.7 & 0.0 & -1.0 & & 69. & 1 & 2.5 & 4.2 \\
\hline 200 & 5 & 108 & 20 & 0.355 & 0.66 & 0.0 & -4.9 & 6. & 65. & 2 . & 14.2 & 4.4 \\
\hline 20080 & 546 & 103 & 20 & 0.371 & 0.65 & 0.15 & -6.7 & 3.4 & 64. & 4.1 & 15.8 & 4.6 \\
\hline 20080 & 467 & 92 & 7 & 0.387 & 0.62 & 0.0 & -11.4 & 3.1 & 62.4 & 2.0 & 14.7 & 4.4 \\
\hline 20080 & & 77 & & & 0.53 & 0.1 & -9.0 & 3.7 & 58.7 & 3. & 48.1 & 4.8 \\
\hline 20080801 & 54680.46382 & 79 & 8 & 0.536 & 0.56 & 0.1 & -10.0 & 3.6 & 62.8 & 3. & 46.3 & 4.6 \\
\hline 20080830 & 54709.46967 & -43 & 8 & 0.013 & 1.11 & 0.0 & -4.2 & 1.1 & 60.6 & 1. & 30.1 & 3.0 \\
\hline 20080922 & 54732.34722 & 95 & 7 & 0.390 & 0.60 & 0.0 & -7.0 & 4.6 & 65. & 3. & 47.2 & 4.7 \\
\hline 20080928 & 54738.39732 & 92 & 10 & 0.490 & 0.63 & 0.0 & -3.0 & 5.0 & 59.3 & 0.9 & 45.6 & 4.5 \\
\hline 20090613 & & 8 & 8 & & 1.05 & 0.0 & -2.1 & 3.4 & 67.0 & 3. & 34.9 & 3.5 \\
\hline 2009 & 071 & -9 & 8 & 0.757 & 23 & 0.1 & -7.2 & 3.3 & 61. & 3.9 & 27.2 & 2.7 \\
\hline & & -15 & 7 & & & & -7.4 & 2.9 & 2 & & 8.4 & 2.8 \\
\hline & & 57 & 7 & & 1.3 & 0.0 & -10.4 & 3. & 55 & & 20.5 & 2.0 \\
\hline 200 & & -51 & $\varepsilon$ & & 1.3 & 0.09 & -9.5 & 3. & 57. & 4 & 21.6 & 2.1 \\
\hline & & -68 & 4 & & 1.3 & 0.1 & -12.3 & 3.5 & 50 & 3. & 9.0 & 1.9 \\
\hline 20090624 & 55007.4 & -77 & 3 & 0.920 & 1.27 & 0.0 & -12.8 & 4.0 & 49. & 5.2 & 21.5 & 2.1 \\
\hline 20090625 & 55007.54341 & -68 & 5 & 0.921 & 1.40 & 0.1 & -13.0 & 3.5 & 53.0 & 3.5 & 20.7 & 2.1 \\
\hline 20090625 & 55008.48148 & -76 & 7 & 0.936 & 1.2 & 0.1 & -9.2 & 3.8 & 56.7 & 3. & 21.7 & 2.2 \\
\hline 20090626 & 55009.48715 & -77 & 7 & 0.953 & 1.2 & 0.1 & -10.1 & 1.9 & 56.5 & 2.7 & 21.3 & 2.1 \\
\hline 20090704 & 55017.36579 & 6 & 8 & 0.083 & 0.82 & 0.08 & -12.0 & 3.1 & 63.6 & 3.4 & 35.3 & 3.5 \\
\hline 20090730 & 55042.51432 & 85 & 7 & 0.497 & 0.61 & 0.07 & -7.5 & 0.5 & 56.5 & 1.0 & 41.5 & 4.1 \\
\hline 20090809 & 55053.35448 & 23 & 8 & 0.675 & 0.85 & 0.07 & -3.6 & 3.6 & 64.3 & 2.5 & 35.1 & 3.5 \\
\hline 20090809 & 55053.39778 & 30 & 7 & 0.676 & 0.85 & 0.07 & -3.7 & 3.8 & 64.3 & 3.6 & 35.5 & 3.5 \\
\hline 20090831 & 55075.41518 & -31 & 7 & 0.038 & 0.90 & 0.08 & -7.3 & 1.9 & 59.0 & 2.3 & 30.3 & 3.0 \\
\hline & & 16 & 8 & 0.087 & 0.86 & & -12.5 & 2.9 & 63.2 & 2.7 & 31.3 & 3.1 \\
\hline 20090907 & 55082.32460 & 55 & 8 & & 0.82 & 0.07 & -10.4 & 4.8 & 68.4 & 2.9 & 34.6 & 3.4 \\
\hline 20090926 & 55101.43383 & 89 & 8 & 0.467 & 0.57 & 0.08 & -8.4 & 3.2 & 61.9 & 1.2 & 45.2 & 4.5 \\
\hline 20090926 & & 93 & 0 & 0.468 & 0.58 & 0.11 & -10.4 & 3.8 & 61.7 & 3.9 & 45.4 & 4.5 \\
\hline 20091004 & & 61 & 8 & 0.597 & 0.66 & 0.12 & -12.6 & 1.8 & 57.2 & 4.2 & 38.4 & 3.8 \\
\hline 20091011 & 55116.39065 & 16 & 8 & 0.713 & 0.91 & 0.07 & -5.9 & 3.9 & 62.6 & 4.3 & 33.1 & 3.3 \\
\hline
\end{tabular}

We find $f(m)=4.1 \pm 0.4 M_{\odot}$ (where the uncertainty includes the errors on $P, k$, and $e$ ) in very good agreement with the value of $4.0 \pm 0.3 M_{\odot}$ that is reported by Richardson et al. (2014). We note that no other reliable parameter for the system components can be obtained without further constraints, such as the detection of spectral features from the secondary component and a very precise photometry.

\subsection{Permitted emission lines}

Permitted metallic emission lines exhibit obvious variability of the blue/red peak ratio (see Fig. 2). Taking into account the possibility of a connection with the absorption lines velocities, we decided to tackle the problem from several points of view. We therefore selected a number of strong and isolated lines of metallic ions. The final list includes: Fe II $\lambda 6456.4, \lambda 6432.7, \lambda 6416.9$, $\lambda 6247.6, \lambda 6084.1, \lambda 5991.4, \lambda 5534.8$, and $\lambda 5425.3 \AA$ which all display a double-peaked or asymmetric profile in all spectra. We note that some Fe II lines were discarded because of either blending with other features, their faintness, or a low $\mathrm{S} / \mathrm{N}$ in the blue spectral range.

First we performed a two-Gaussian fit to each line to measure the intensity and wavelength of the blue and red peaks. The results are presented in Table 1 . We found a modulation of the 
A\&A 585, A60 (2016)
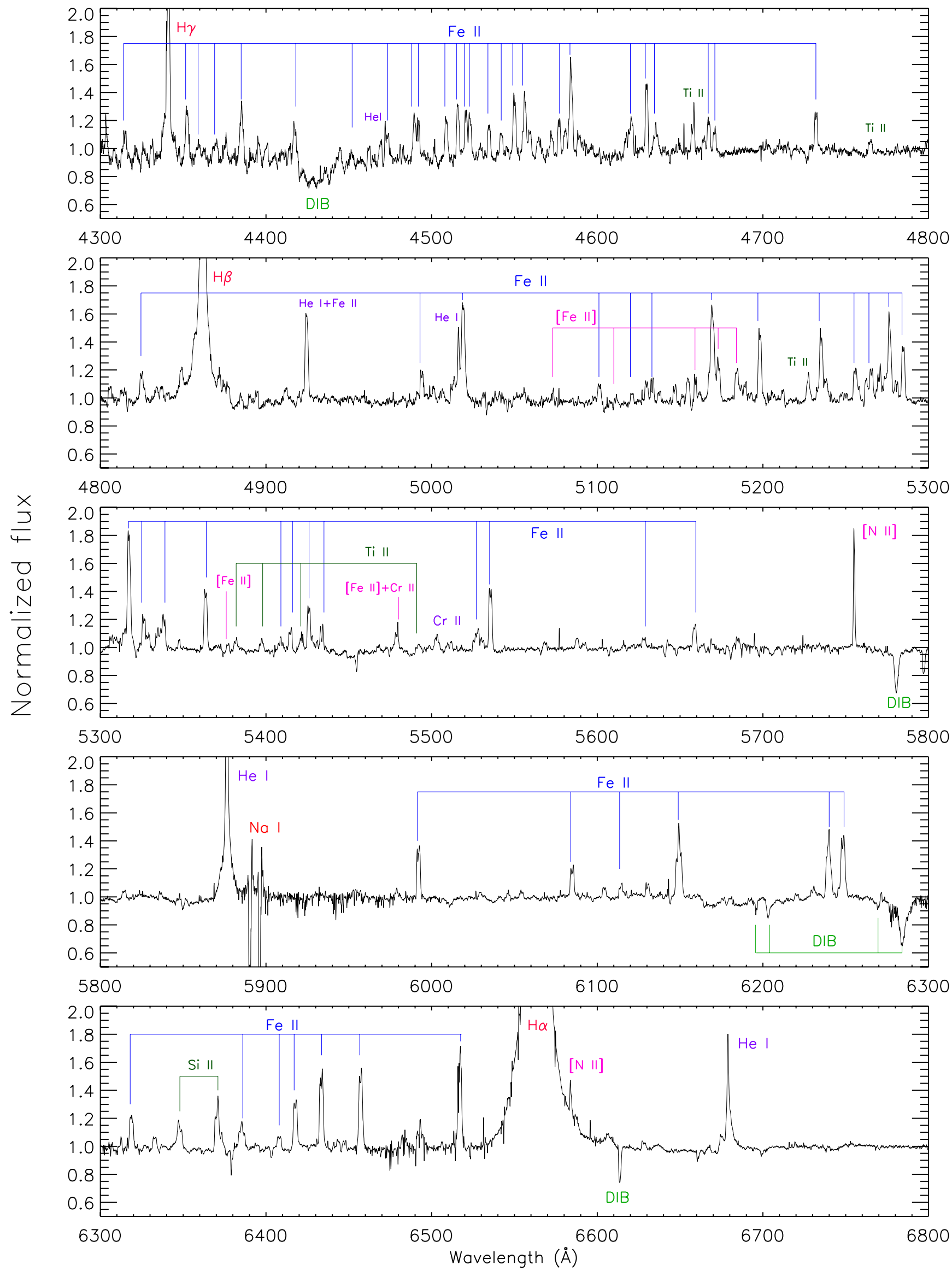

Fig. 1. Continuum-normalized ElodIE spectrum, taken in August 1995, with the most prominent emission and absorption lines identified. All the absorption lines visible between the Na I D doublet and $6000 \AA$ and those on the blue side of the H $\alpha$ line have a telluric origin. 


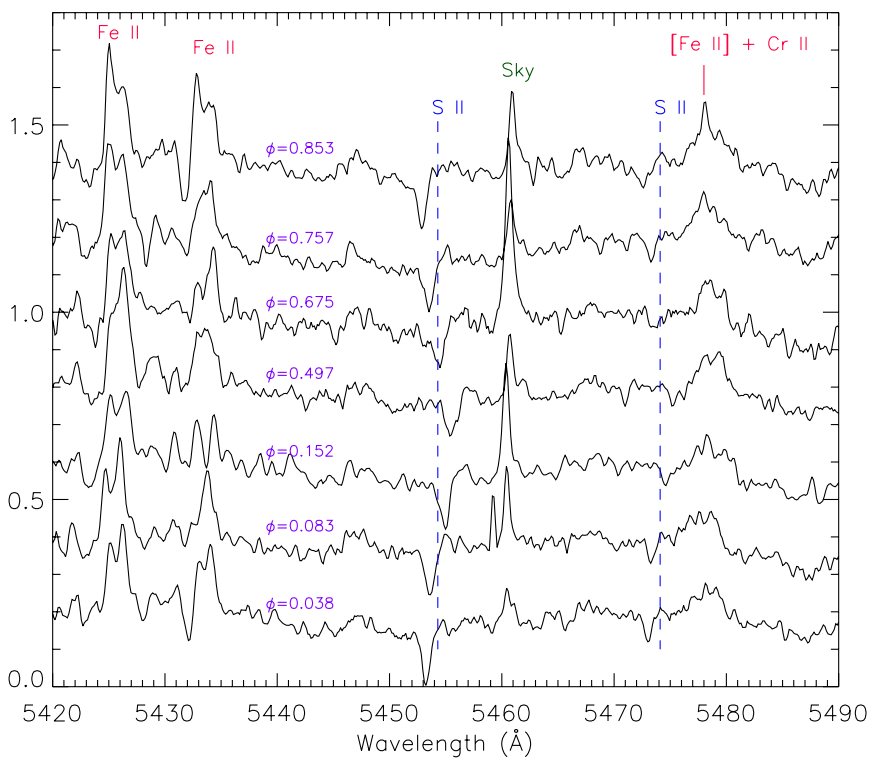

Fig. 2. Example of seven FRESCO spectra in the range around $5450 \AA$. The velocity shift of the absorption lines (S II $\lambda 5453.8$ and $\lambda 5473.6 \AA$ ) is apparent. Also note the variable intensity ratio of the blue and red peaks of the emission line Fe II $\lambda 25425.3 \AA$.

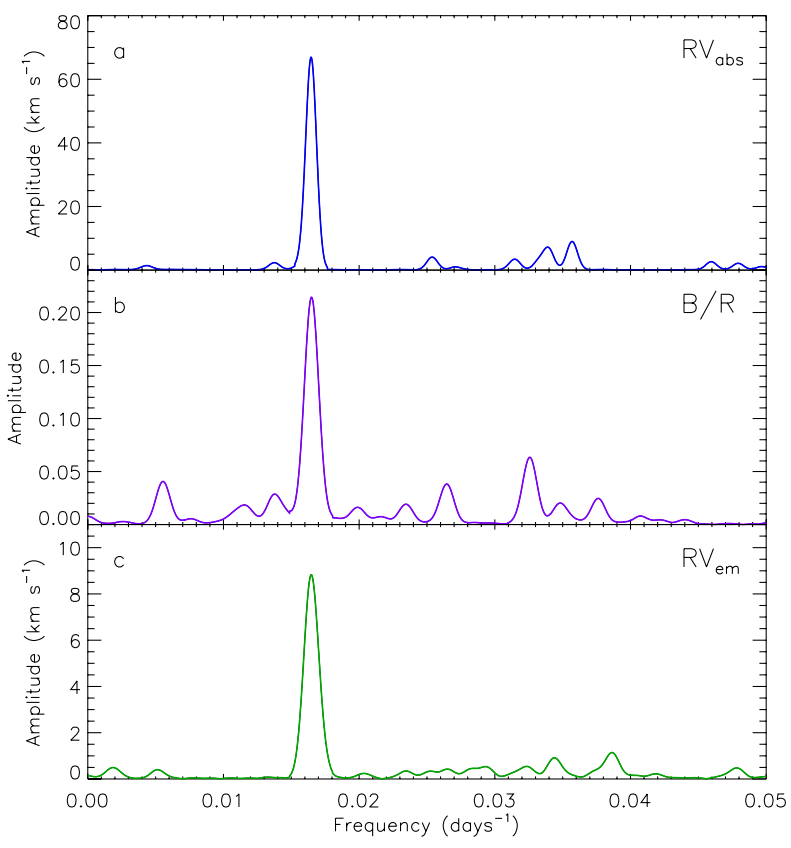

Fig. 3. Cleaned periodograms for $\mathrm{RV}_{\mathrm{abs}}$ (top panel), $B / R$ peak ratio (middle panel), and $\mathrm{RV}_{\mathrm{em}}$ (lower panel). The 60-day period is clearly evident in all diagrams.

blue/red peak intensity ratio $(B / R)$ with the same period as that of $\mathrm{RV}_{\mathrm{abs}}$. Indeed, the CLEAN analysis performed on these ratios gives a period of $60.6 \pm 2.2$ days (see Fig. 3 ) which is the same as that of $\mathrm{RV}_{\mathrm{abs}}$ within the uncertainties. The $B / R$ curve and the $\mathrm{RV}_{\mathrm{abs}}$ one are nearly anti-correlated, as can be seen in Fig. 4. The shape of the two curves is slightly different: the minimum of $B / R$ is observed later than the maximum of $\mathrm{RV}_{\mathrm{abs}}$, while the largest $B / R$ ratios are observed just before the minimum of $\mathrm{RV}_{\mathrm{abs}}$. The high degree of correlation of the two curves is demonstrated by a very high rank-correlation (Press et al. 1986) coefficient $\rho=-0.82$.

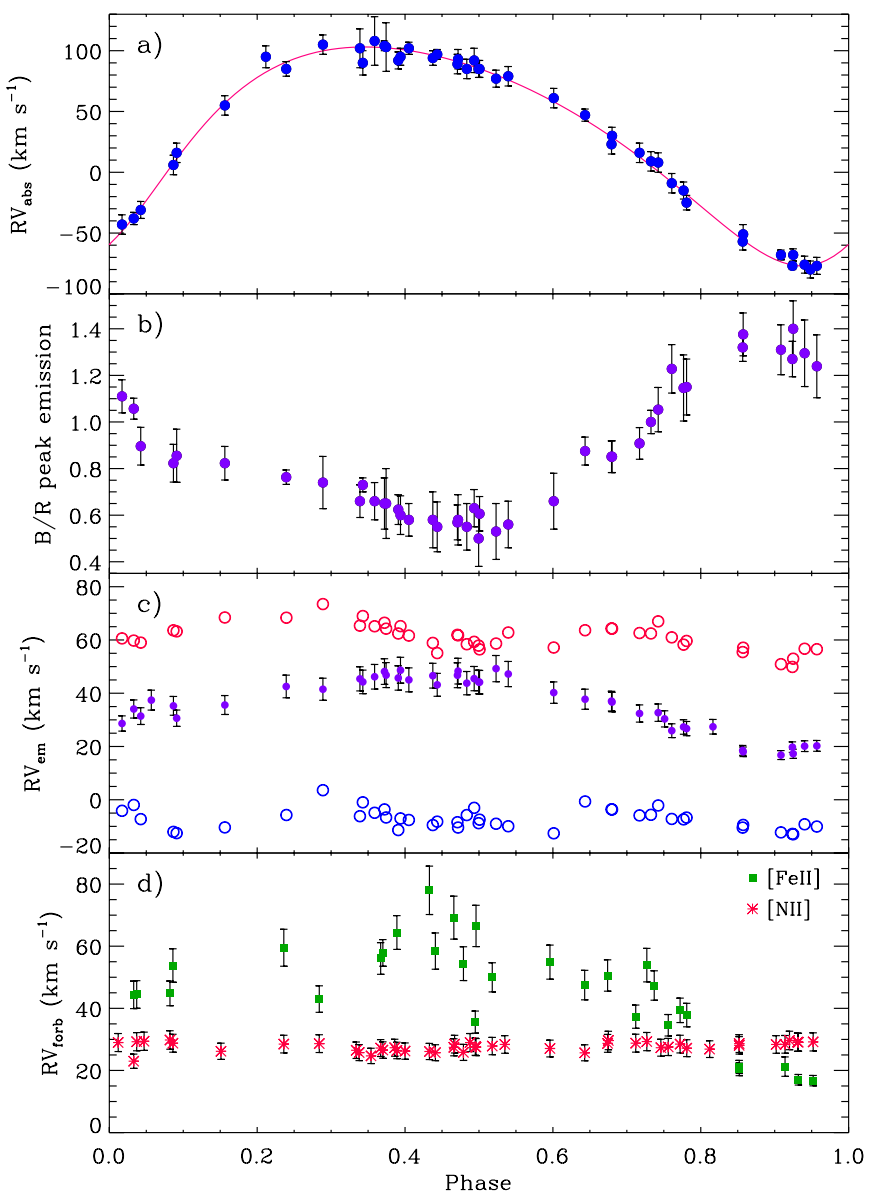

Fig. 4. From top to bottom: a) radial velocity curve of MWC 314 obtained with the absorption lines (dots), with the RV solution overplotted as a continuous line. b) The intensity ratio of the blue to the red peak of the Fe II emission lines. c) Radial velocity of the Fe II emission lines as measured from their centroids (filled dots) and as evaluated from the red and blue emission peaks (upper and lower empty circles, $\mathrm{RV}_{\mathrm{em}}^{\mathrm{R}}$ and $\mathrm{RV}_{\mathrm{em}}^{\mathrm{B}}$ ). d) Radial velocity of the forbidden [Fe II] and [N II] lines.

We also plotted the blue and red peak RVs individually. The situation is much more complicated here. Sometimes the lines are only asymmetric with one of the peaks missing. In other cases more than two peaks are present simultaneously in all the lines, which makes the measurement much more uncertain. The results are presented in Table 1 (Cols. 8 to 11) and in Fig. 4c. These RVs show small-amplitude scattered variations with only a possible modulation with the orbital 60-day period. However, the velocity difference seems to be constant except for a few cases around phase 0.15 (see bottom panel of Fig. 6).

Given the unclear situation for the RV of the double peaks, we decided to additionally measure the equivalent width (EW) of the emission lines and the centroid of each line, without fitting its peaks but treating it as a single feature. To do this, we used the SPLOT task in IRAF. The RVs, calculated from the line centroids, are listed in Table $1\left(\mathrm{RV}_{\mathrm{em}}^{\mathrm{S}}\right.$, Cols. 12 and 13) and displayed in Figs. 4c and 6a. A clear, positive correlation with the RV curve of the absorption lines is now evident, although with an amplitude smaller than that of $\mathrm{RV}_{\mathrm{abs}}$ and $\mathrm{RV}$ s ranging from +15 to $+50 \mathrm{~km} \mathrm{~s}^{-1}$. The periodogram analysis applied to the RV of the emission lines practically gives the same period as for the absorptions. The fit of the $\mathrm{RV}_{\mathrm{em}}^{\mathrm{S}}$ curve with an eccentric orbit provides the values reported in Table 2 . This solution is shown as a full line in Fig. 6a. The cross-correlation of $\mathrm{RV}_{\mathrm{em}}^{\mathrm{S}}$ and 
Table 2. Orbital parameters derived from the RV of the absorption and emission lines.

\begin{tabular}{lllllll}
\hline \hline $\begin{array}{l}P_{\text {orb }} \\
\text { (days) }\end{array}$ & $\begin{array}{l}\text { HJD } \\
(-2400000)\end{array}$ & $e$ & $\omega$ & $\begin{array}{l}\gamma \\
\left(\mathrm{km} \mathrm{s}^{-1}\right)\end{array}$ & $\begin{array}{l}k \\
\left(\mathrm{~km} \mathrm{~s}^{-1}\right)\end{array}$ & Diagnostics/Ref. \\
\hline $60.737(0.008)$ & $49546.01(1.10)$ & $0.244(0.020)$ & $218.7^{\circ}(5.7)$ & $30.7(1.3)$ & $89.7(2.0)$ & Absorption lines \\
$60.74(0.03)$ & $49546(2)$ & $0.25(0.05)$ & $170^{\circ}(10)$ & $35.0(1.0)$ & $13.5(1.0)$ & Fe II emission lines \\
$60.799977(0.000014)$ & $54959.76(0.56)$ & $0.235(0.003)$ & $289^{\circ}(1)$ & $28.44(0.17)$ & 84.5 & Lobel et al. (2013) \\
$60.735(0.024)$ & $55618.49(0.65)$ & $0.29(0.02)$ & $206.4^{\circ}(4.2)$ & $31.3(1.1)$ & $89.6(1.8)$ & Richardson et al. (2014) \\
\hline
\end{tabular}

Notes. Standard errors are reported in parentheses.

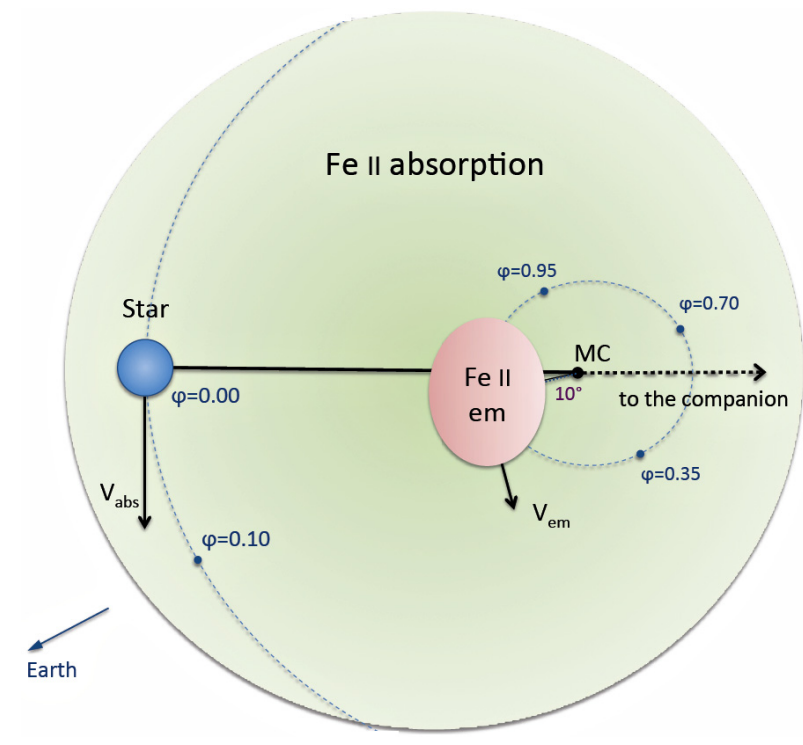

Fig. 5. Sketch of the system geometry with the emitting and absorbing Fe II regions. The orbit of the star and that of the corotating Fe II emitting region are depicted with dotted lines and the phases of conjunctions and quadratures are also indicated. The size of the star and the emitting region are not to scale.

$\mathrm{RV}_{\mathrm{abs}}$ indicates that the former precedes the latter by a very small (and likely insignificant) phase shift $\Delta \phi \approx-0.03$ (corresponding to $\approx 10^{\circ}$ ).

The same orbital period and the very similar $\gamma$ velocity suggest that the bulk of the emission is coming from a region corotating with the binary system but much closer to the center of mass (MC) than the source of the absorption lines. A simple geometrical sketch of the source of the Fe II emission is depicted in Fig. 5.

With this simple geometrical model we also tried to understand the orbital modulation of the $B / R$ peak ratio. We propose a hypothesis that the double-peaked shape of the permitted Fe II emission lines is a result of an extra-absorption that overlaps a pure emission profile. If the two profiles have different full width at half maximum (FWHM) and velocities, a changing blue/red peak asymmetry in phase with the orbit can be reproduced. The continuous line that is overplotted on the observed $B / R$ curve in Fig. $6 \mathrm{~b}$ is derived from the synthetic double-peaked profiles, obtained by the superposition of a broad emission (a Gaussian with $F W H M=115 \mathrm{~km} \mathrm{~s}^{-1}$ ), which moves according to the $\mathrm{RV}_{\mathrm{em}}^{\mathrm{S}}$ curve (full line in Fig. 6a), and a narrower absorption Gaussian $\left(F W H M=25 \mathrm{~km} \mathrm{~s}^{-1}\right)$ at the MC velocity.

As indicated by the $\mathrm{RV}_{\mathrm{em}}^{\mathrm{S}}$ semi-amplitude of only $13.5 \mathrm{~km} \mathrm{~s}^{-1}$, the emitting region must be rather compact (or centrally condensed) and should be located much closer to the MC than the star which is the source of the observed
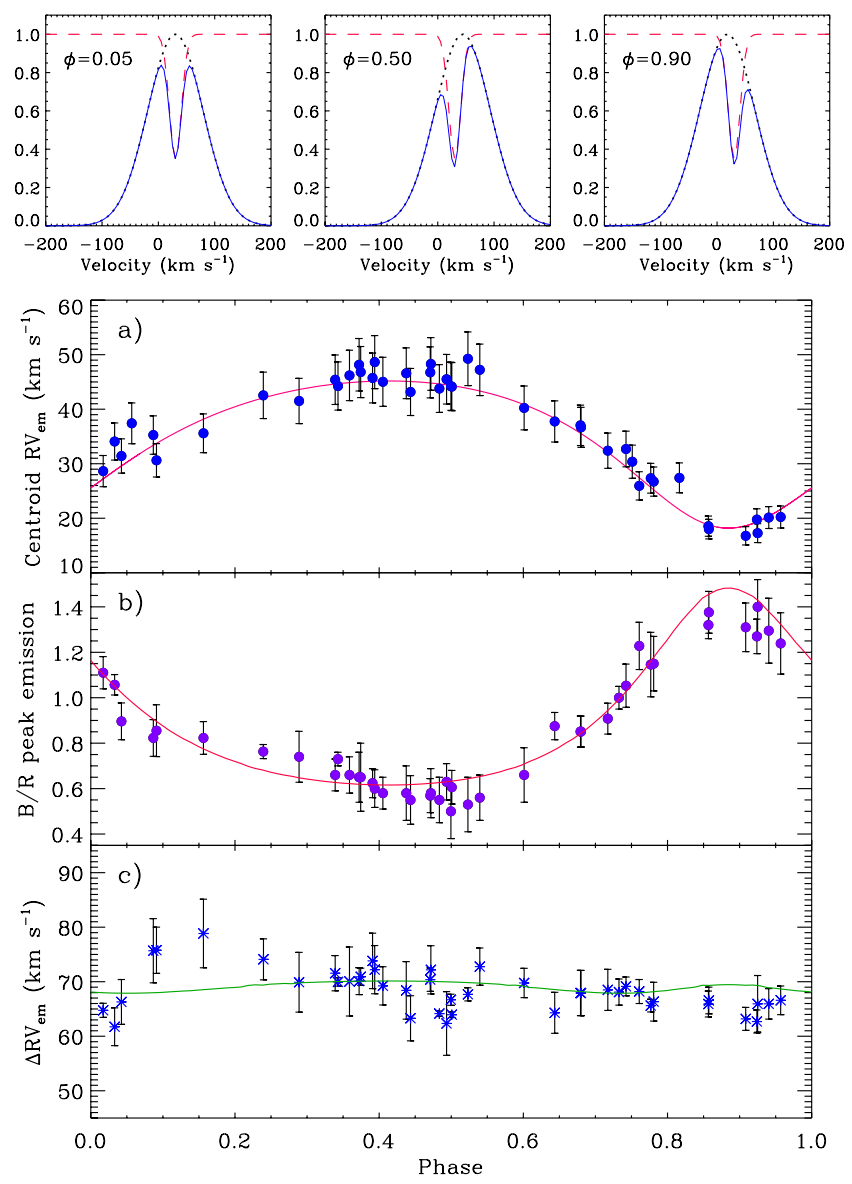

Fig. 6. Upper three panels: synthetic Fe II line profiles (full lines), at three orbital phases $(\phi)$, resulting from the superposition of a "moving" broad Gaussian emission (dotted lines) and a "static" narrower Gaussian absorption. Lower panels: radial velocity $\mathrm{RV}_{\mathrm{em}}^{\mathrm{S}}$ a), and $B / R$ intensity ratio b) of the Fe II emission lines (dots) with the result of the geometrical model superimposed with full lines. Panel c): the velocity separation (asterisks) of the red-blue peaks. $\left(\mathrm{RV}_{\mathrm{em}}^{\mathrm{R}}-\mathrm{RV}_{\mathrm{em}}^{\mathrm{B}}\right)$ with the result of our model superimposed with a full line.

absorption lines. As a simple speculation, if the location of this emitting region is close to the Lagrangian point $\mathrm{L}_{1}$, one may suggest that it is related to a denser part of the stellar wind that is funneled through $\mathrm{L}_{1}$ towards the unseen companion. Conversely, the excess absorption shows a constant intensity and velocity, so it must be caused by a quite large and homogeneous structure, which is always projected onto the emission produced by the compact region. Hence it could be argued that the source of the excess absorption is a circumstellar envelope or shell.

Although this is a simplified model, it allows us to explain the observed behavior of the permitted Fe II emission lines and could be taken as a starting point for more sophisticated models. 
The EWs of the measured emission lines show no evidence for periodicity. This supports the hypothesis of a purely "geometrical" effect produced by a static and nearly homogeneous absorbing envelope over the compact emitting region.

\subsection{Forbidden emission lines}

Similarly to the permitted emission lines, metallic forbidden lines have double-peaked profiles that are not always well structured. Three peaks are clearly present in some spectra. Most of them are blended with other emissions, so that we could not obtain definite results. The final list of [Fe II] lines to analyze only includes $\lambda$ 5627.5, $\lambda$ 5376.5, and $\lambda 5158.8 \AA$.

As already described in Muratorio et al. (2008), forbidden lines are narrower than permitted lines: a mean FWHM of $55 \pm 8 \mathrm{~km} \mathrm{~s}^{-1}$ is found from a single Gaussian fitting. The RV curve reproduces the behavior of the permitted (and therefore of the absorption) lines though with quite a large scatter, likely the result of the small number of lines and their faintness (see Fig. 4d). Surprisingly, the RV variation of the [Fe II] lines is larger than that of the permitted lines, indicating a different emission region. In a very simplified scheme of matter corotating with the system, this would mean that this region is located farther from the MC of the system towards the star, which is the source of the absorption lines. However, this result needs to be confirmed by further observations with a higher $\mathrm{S} / \mathrm{N}$.

An interesting case is the [N II] $5754.59 \AA$ line, which shows a clear double-peaked profile in all the ELODIE spectra $(R=$ 42000 ), where the red peak is always fainter than the blue one, as was also found by Lobel et al. (2013). The mean RV difference between the peaks is $27.5 \pm 1.0 \mathrm{~km} \mathrm{~s}^{-1}$ (see Fig. 7), which is much lower than the peak separation of $\sim 70 \mathrm{~km} \mathrm{~s}^{-1}$ measured for the permitted emission lines (see Fig. $4 \mathrm{c}$ ). The $B / R$ peak ratio ranges from 1.3 to 1.6. The other spectra have a lower resolution that does not permit the peaks to be separated, however the FRESCO spectra indicate an asymmetry of the blue/red side at the top of the line, which is never reversed. Other parameters characterizing this line remained constant during the long period of our observations. The results can be summarized as follows: $F W H M=50 \pm 5 \mathrm{~km} \mathrm{~s}^{-1}, E W=0.8 \pm 0.1 \AA$, and $\langle R V\rangle=28.0 \pm 1.3 \mathrm{~km} \mathrm{~s}^{-1}$. The latter is very close to the $30 \mathrm{~km} \mathrm{~s}^{-1}$ of the barycentric velocity (see Fig. 4). This could indicate an emitting region located around the MC or, most likely, an emitting envelope and/or shell with a large extension, which encompasses the entire binary system, and which is similar to the one that we assumed as giving rise to the narrow Fe II absorption. A very similar behavior is displayed by the [N II] $6583.45 \AA$ line.

With this data, we cannot say whether the extra absorption is a feature related to the formation of the [N II] lines or if it is an effect of the same cloud that causes the excess absorption in the permitted Fe II lines. In the latter hypothesis, the absence of inversion of the $\mathrm{B} / \mathrm{R}$ peak ratio is due to the stability of [N II] line RV.

However, this line can mainly form in the large bipolar outflow around MWC 314 which has been discovered by Marston \& McCollum (2008), using narrow-band $\mathrm{H} \alpha$ imaging. Since both the ELODIE and FRESCO spectra are taken with optical fibers that encompass a few arcseconds on the sky around the central star, the double-peaked profiles could be indicative of the approaching and receding parts of the outflow in the line of sight of the central star. Similar [N II] $6583.45 \AA$ double-peaked profiles with velocity separations of the same order have been also observed in LBV candidates in the Large Magellanic Cloud by Weis (2003).

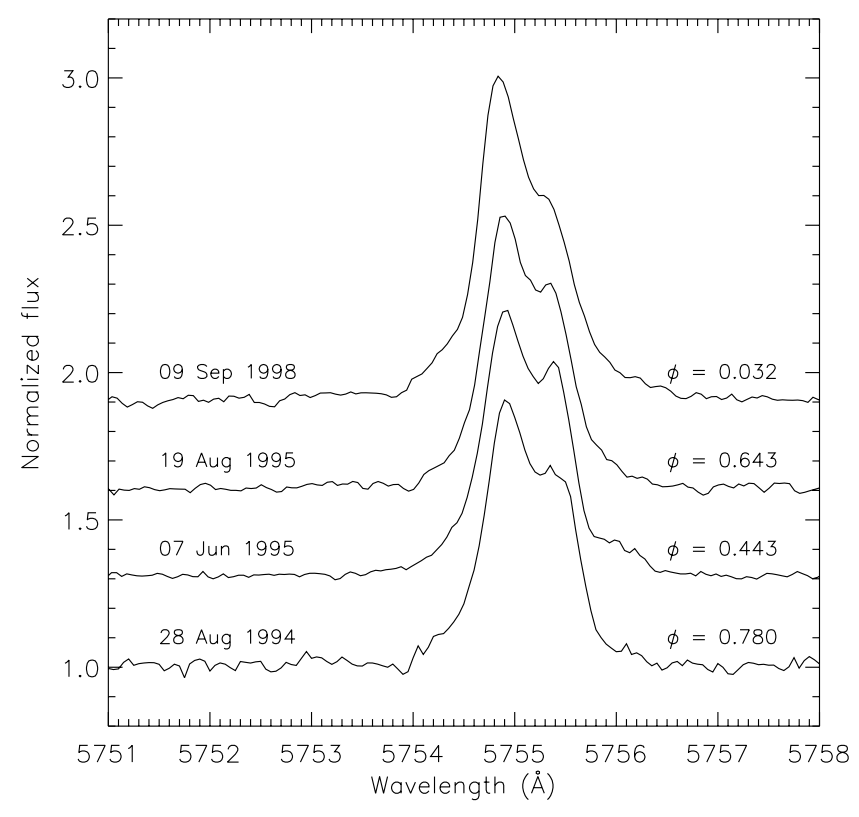

Fig. 7. The [N II] $5754.59 \AA$ line profiles of the OHP spectra; the observed spectra are displayed in a heliocentric wavelength scale.

\section{Hydrogen and helium line profiles}

We also investigated the behavior of the helium and hydrogen lines with the orbital phase.

The $\mathrm{H} \alpha$ line with a peak intensity $\sim 20$ in the continuum units and a full width at zero level of $\sim 70 \AA$ is a strong and broad feature. It is difficult to set the continuum level around the line and precisely measure its EW. However, its centroid (and RV) can be defined with sufficient accuracy. Most of the other $\mathrm{H}$ and $\mathrm{He}$ lines are either faint, fall near edges of échelle orders, or are blended with other emission lines. We found that the He I $\lambda 5876 \AA$ and $\mathrm{H} \beta$ lines appear in all our spectra, are strong enough, and are unsaturated. Unfortunately, the other strong helium line in the optical range, He I $\lambda 6678 \AA$, falls in the gap between the last two échelle orders in our FRESCO spectra. In the same spectra, $\mathrm{H} \gamma$ falls near the edges of two orders, but we could measure its EW and RV in the best exposed ones.

The EWs of $\mathrm{H} \alpha, \mathrm{H} \beta$, and $\mathrm{H} \gamma$ are in the ranges of $110-140 \AA$, 12-17 $\AA$, and 2-5 lation. The same holds true for their RVs which are scattered between $\approx 30$ and $45 \mathrm{~km} \mathrm{~s}^{-1}$. The absence of orbital modulation in both the EWs and RVs of the Balmer lines suggests that these features are neither related to one of the stellar components nor are they formed in a compact source corotating with the system, such as the source of Fe II lines. The Balmer lines, more likely, originate in a large region or in different regions that occupy a large portion of the circumbinary space.

The most interesting behavior shows the profile of the He I $\lambda 5876$ line, which changes from a nearly symmetric emission to a P-Cygni profile. This is clearly illustrated by the two OHP-ELODIE spectra shown in Fig. 8. The blue-shifted extraabsorption visible in the spectrum at the phase $\phi=0.443$ seems to be a result of different components with velocities from $\sim-200 \mathrm{~km} \mathrm{~s}^{-1}$ to $\leq-1000 \mathrm{~km} \mathrm{~s}^{-1}$.

To check if this feature has a random occurrence or whether it is related to particular orbital configurations, we produced a trailed spectral image with all the FRESCO and Asiago spectra in the region around the $\mathrm{He} \mathrm{I} \lambda 5876 \AA$ line (Fig. 9). It clearly shows that the extra absorption is mainly observed at phases 


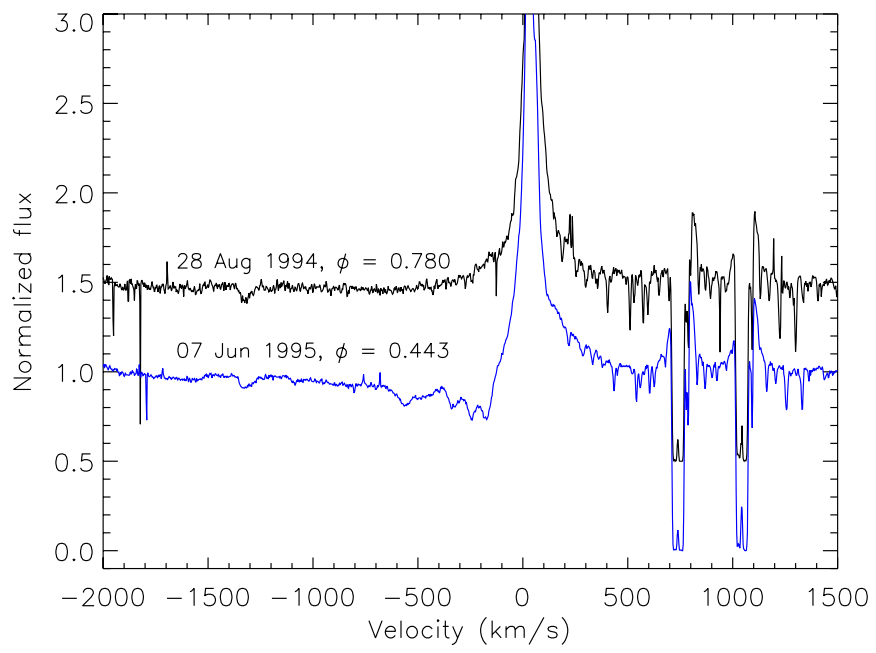

Fig. 8. OHP-ELODIE spectra in the He I region plotted in a scale of heliocentric velocity. The spectra are vertically shifted for the sake of clarity.

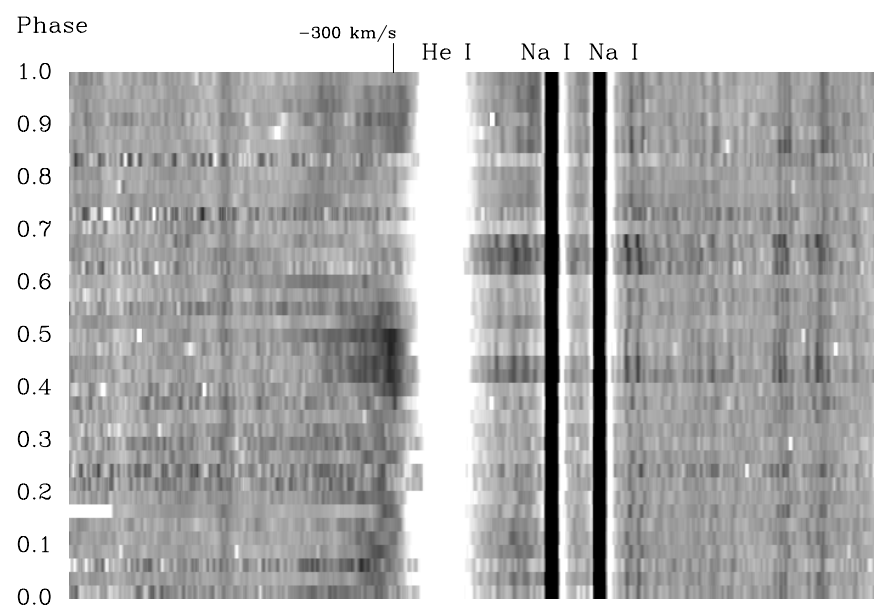

Fig. 9. Trailed spectrum in the He I $\lambda 5876$ region produced with the FRESCO and Asiago spectra. The He I emission peak has been artificially saturated to emphasize the extra-absorption feature.

between 0.40 and 0.55 . Although with a smaller intensity, some extra-absorption is also observed at phases around $\phi=0.9$ and $\phi=0.1$ (primary star in front). This is the configuration where Lobel et al. (2014) observed this phenomenon. They ascribed it to wave propagation linked to the orbital motion near the lowvelocity regions of the stellar wind that are close to the primary star. Note that, because of the different ephemeris, the phases in Lobel et al. (2014) are shifted by $\sim-0.15$ compared to ours. They suggest that these absorption features can also be originated in dynamical wind regions that are confined between the two components. Our observations indicate that these extra absorptions are stronger when the primary star is behind the "interbinary" wind. This can be understood if the primary component is filling (or almost filling) its Roche lobe, as proposed by Lobel et al. (2013) and Richardson et al. (2014), and transferring mass to the secondary through Roche lobe overflow. The strengthening of the extra absorption at these phases could be the result of a gas stream projected over the primary star or to overdense regions in the stellar wind, which is funneled through $\mathrm{L}_{1}$. Thus, the source of this phenomenon could be different, both physically and geometrically, than the one that is originating the
Fe II emission. This is in line with the detailed model of asymmetric wind developed by Lobel et al. (2013). A detailed physical model of the He I profile is, however, beyond the scope of the present paper.

\section{Concluding remarks}

The large number of high resolution spectra, which span nearly 15 years, has allowed us to confirm the binary nature of MWC 314 and provide a very accurate solution for the RV curve obtained from the stellar absorption lines.

The numerous metallic emission lines present in the spectrum very often display double-peaked profiles with a variable shape. The RV variations of the permitted Fe II emission lines, as derived from their centroids, are clearly periodic and in phase with the RV curve of the star that produces the absorption spectrum, although with a much smaller RV semi-amplitude. The blue/red peak ratio is also strongly correlated with the orbital period.

We proposed a simple geometrical model, where the source of Fe II emission is rather compact and located near the system MC that is possibly close to the Lagrangian $\mathrm{L}_{1}$ point. In this model, the central absorption in the Fe II lines forms in a very wide (circumbinary) region that slowly rotates around the MC (see Fig. 5) and is is always projected over the source of emission. This model allows us to explain the observed variation of both the radial velocity $\left(\mathrm{RV}_{\mathrm{em}}^{\mathrm{S}}\right)$ and the $B / R$ peak ratio of the permitted Fe II lines fairly well, and is compatible with a more sophisticated 3D wind model proposed by Lobel et al. (2012).

The optically thin [Fe II] lines also display double-peaked profiles but their RV variation has a larger amplitude compared with the Fe II ones, indicating that these lines are formed closer to the primary star and possibly in a circumstellar disk. However since the [Fe II] lines are weaker than those of Fe II, this result needs further verification using higher $\mathrm{S} / \mathrm{N}$ spectra.

Other emission lines, such as the [N II] $5754 \AA$ and the Balmer lines have an almost constant RV, which is nearly coincident with the $\gamma$ velocity. They likely originate in a distant, circumbinary region, and possibly in the inner parts of the large bipolar outflow discovered by Marston \& McCollum (2008) using narrow-band $\mathrm{H} \alpha$ imaging.

The most prominent variable feature observed in the He I $\lambda 5876 \AA$ Aline profile is a broad blue-shifted absorption that is mostly observed at phases between 0.40 and 0.55 (primary star behind). This is likely the result of the wind of the primary component, funneled towards the companion, which is interposed between us and the primary star in these phases.

To continue revealing the nature of the MWC 314 binary system, is needed a new set of high-resolution and high $\mathrm{S} / \mathrm{N}$ spectra. This would cover the orbital phases in depth and focus on weak spectral lines, in an attempt to get information about the region where forbidden lines form and to help search for the elusive secondary companion. Contemporaneous multicolor photometry will help to associate spectral and brightness variations.

Acknowledgements. The authors thank the staff of the OAC for their technical support during the observations; IRAF is distributed by the NOAO, which is operated by AURA under contract with NFS. This project was partially supported by the INAF grant 2007 "Luminous Blue Variable phenomenon: towards a better understanding of massive stars evolution". We thank the anonymous referee for useful comments and suggestions.

\section{References}

Bevington, P. R. 1969, Data Reduction and Error Analysis for the Physical Sciences (McGraw-Hill Book Company), 237 
A. Frasca et al.: The spectral behavior of MWC 314

Glatzel, W., \& Chernigovski, S. 2001, in P Cygni 2000, 400 Years of Progress, eds. M. de Groot, \& C. Sterken, ASP Conf. Ser., 233, 227

Lobel, A., Groh, J., Torres Dozinel, K., et al. 2012, in Four Decades of Massive Star Research - A Scientific Meeting in Honor of A. F. J. Moffat, eds. L. Drissen, C. Robert, \& N. St.-Louis, ASP Conf. Ser., 465, 358

Lobel, A., Groh, J.H., Martayan, C., et al. 2013, A\&A, 559, A16

Lobel, A., Martayan, C., Corcoran, M., Groh, J. H., \& Frémat, Y. 2014, in New windows on massive stars: asteroseismology, interferometry, and spectropolarimetry, Proc. IAU Symp., 307, 115

Marston, A. P., \& McCollum, B. 2008, A\&A, 477, 193

Miroshnichenko, A. S. 1996, A\&A, 312, 941

Miroshnichenko, A. S., Fremát, Y., \& Hauziaux, L. 1998, A\&A, 131, 469

Muratorio, G., Rossi, C., \& Friedjung, M. 2008, A\&A, 637, 64
Nota, A., Livio, M., Clampin, M., \& Shulte-Ladbeck, R. 1995, A\&A, 448, 788 Press, W. H., Flannery, B. P., Teukolsky, S. A., \& Vetterling, W. T. 1986, Numerical Recipes, The Art of Scientific Computing (Cambridge University Press), 489

Richardson, N. D., Moffatt, A. F. J., Maltais-Tariant, R., et al. 2014, SPIE Proc., 9146, 91460G-1

Roberts, D. H., Lehar, J., \& Dreher, J. W. 1987, AJ, 93, 968

Rossi, C., Frasca, A., Marilli, E., et al. 2011, in Active OB stars: structure, evolution, mass loss and critical limits, eds. C. Neiner, G. Wade, G. Meynet, \& G. Peters, IAU Symp., 272, 422

Scargle, J. D. 1982, ApJ, 263, 835

Weis, K. 2003, A\&A, 408, 205

Wisniewski, J. P., Babler, B. L., Bjorkman, K. S., et al. 2006, PASP, 118, 820 